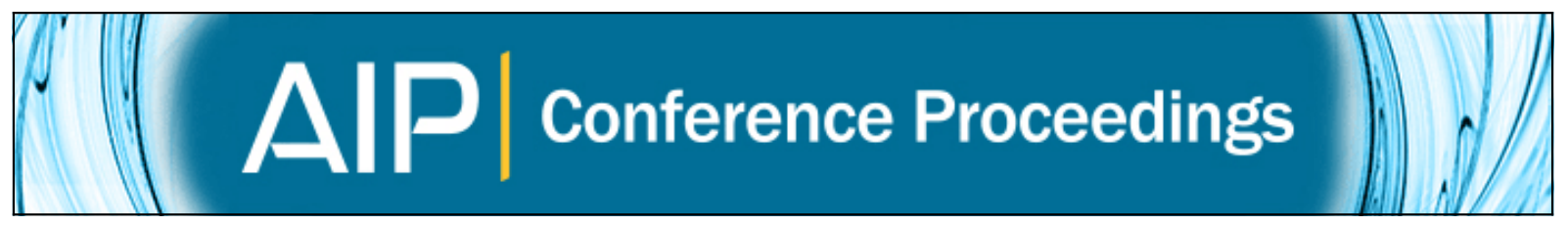

\title{
Analytical solution of two-phase spherical Stefan problem by heat polynomials and integral error functions
}

Stanislav N. Kharin, Merey M. Sarsengeldin, and Hassan Nouri

Citation: AIP Conference Proceedings 1759, 020031 (2016); doi: 10.1063/1.4959645

View online: http://dx.doi.org/10.1063/1.4959645

View Table of Contents: http://scitation.aip.org/content/aip/proceeding/aipcp/1759?ver=pdfcov

Published by the AIP Publishing

\section{Articles you may be interested in}

Exact solution of two phase spherical Stefan problem with two free boundaries

AIP Conf. Proc. 1759, 020117 (2016); 10.1063/1.4959731

Numerical study of heat transfer problems in two-phase flows involving temperature distribution within dispersed solid particles

AIP Conf. Proc. 1702, 190012 (2015); 10.1063/1.4938979

Some explicit solutions for a class of one-phase Stefan problems

AIP Conf. Proc. 1479, 2379 (2012); 10.1063/1.4756673

Numerical solution of one-phase Stefan problem for a non-classical heat equation

AIP Conf. Proc. 1293, 39 (2010); 10.1063/1.3515601

Analytic Solutions to Two Albedo Problems

J. Math. Phys. 11, 1735 (1970); 10.1063/1.1665319 


\title{
Analytical solution of two-phase spherical Stefan problem by heat polynomials and integral error functions
}

\author{
Stanislav N. Kharin", , Merey M. Sarsengeldin" ${ }^{* \dagger}$ and Hassan Nouri* \\ *Institute of Mathematics and Mathematical Modeling, 050010, Almaty, Kazakhstan \\ ${ }^{\dagger}$ Kazakh-British Technical University, Almaty, Kazakhstan
}

\begin{abstract}
On the base of the Holm model, we represent two phase spherical Stefan problem and its analytical solution, which can serve as a mathematical model for diverse thermo-physical phenomena in electrical contacts. Suggested solution is obtained from integral error function and its properties which are represented in the form of series whose coefficients have to be determined. Convergence of solution series is proved.
\end{abstract}

Keywords: Thomson effect, Symmetric and asymmetric contacts

PACS: $51.30 .+\mathrm{i}, 64.70 . \mathrm{dj}$

\section{INTRODUCTION}

In most electric contacts with small contact surface $\left(b<10^{-4}\right)$ and low electric current it is sufficient to use Holm's ideal sphere [1] for investigation of diverse thermo-physical phenomena in electric contacts. Such processes like arcing and bridging are so fleeting (nana second range) that their experimental study is very difficult or sometimes impossible and the need of modeling is due not only to the need to optimize the planning experiment, but also due to the impossibility to use a different approach. So called Stefan type problems which take in account phase transformations, agree with experimental data [2] and can serve as a model for afore mentioned processes [3-5]. From theoretical point of view, these problems are among the most challenging problems in the theory of non-linear parabolic equations, which along with the desired solution an unknown moving boundary has to be found. In some specific cases it is possible to construct Heat potentials for which, boundary value problems can be reduced to integral equations $[3,4,6]$. However, in the case of domains that degenerate at the initial time, there are additional difficulties because of the singularity of integral equations, which belong to the class of pseudo - Volterra equations which are unsolvable in the general case. First attempts to solve Stefan problem by proposed method are given in [7, 8]. This study is devoted to suggest analytical solution for two phase spherical Stefan problem with one free boundary which is based on the use of integral error functions and their properties.

\section{TWO PHASE SPHERICAL STEFAN PROBLEM}

\section{Preliminaries}

1. Complementary error function is represented as following:

$$
i^{n} \operatorname{erfcx}=\frac{2}{\sqrt{\pi}} \frac{1}{n !} \int_{x}^{\infty}(v-x)^{n} \exp \left(-v^{2}\right) d v .
$$

Lemma 1 By L'Hopital's rule it is not difficult to show that

$$
\lim _{x \rightarrow \infty} \frac{i^{n} \operatorname{erfc}(-x)}{x^{n}}=\frac{2}{n !},
$$

and

$$
\lim _{t \rightarrow 0}(2 a \sqrt{t})^{n} i^{n} \operatorname{erfc}\left(-\frac{x}{2 a \sqrt{t}}\right)=\frac{2}{n !} x^{n} .
$$

\footnotetext{
International Conference on Analysis and Applied Mathematics (ICAAM 2016)

AIP Conf. Proc. 1759, 020031-1-020031-6; doi: 10.1063/1.4959645

Published by AIP Publishing. 978-0-7354-1417-4/\$30.00
} 


\section{Analytical solution}

Let's consider two-phase Stephan Problem, which enables to describe heat transfer in electrical contacts. The heat flux $P(t)$ entering in the sphere of the radius $b$ melts contact material (liquid zone $b<r<\alpha(t)$ ) and passes further through the solid zone $\alpha(t)<r<\infty$.

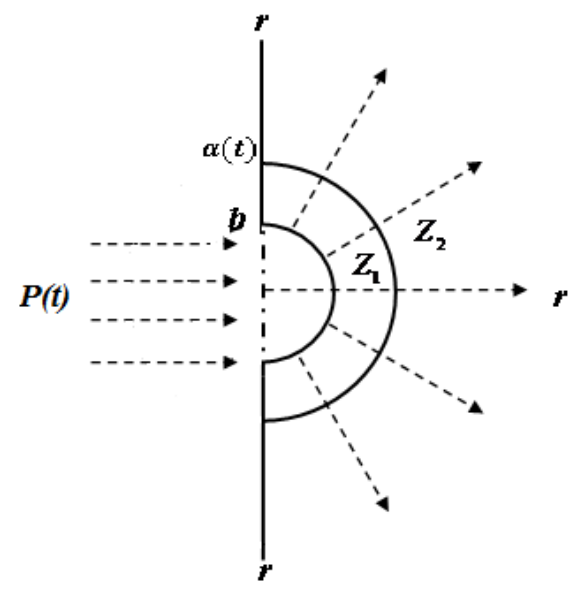

FIGURE 1. Temperature distribution in electric contact. Holm hemisphere

The heat equations for each zone are as follows:

$$
\begin{aligned}
\frac{\partial \theta_{1}}{\partial t} & =a_{1}^{2}\left(\frac{\partial^{2} \theta_{1}}{\partial r^{2}}+\frac{2}{r} \frac{\partial \theta_{1}}{\theta r}\right), \quad b<r<\alpha(t), \\
\frac{\partial \theta_{2}}{\partial t} & =a_{2}^{2}\left(\frac{\partial^{2} \theta_{2}}{\partial r^{2}}+\frac{2}{r} \frac{\partial \theta_{2}}{\theta r}\right), \quad \alpha(t)<r<\infty, \\
\theta_{1}(b, 0) & =T_{m}, \\
\theta_{2}(r, 0) & =f(r), \\
f(b) & =T_{m}, \quad \alpha(0)=b, \quad f(\infty)=0, \\
r & =b: \quad-\lambda_{1}=\frac{\partial \theta_{1}(b, t)}{\partial r}=P(t), \\
r & =\alpha(t): \quad \theta_{1}(\alpha(t), t)=T_{m}, \\
\theta_{2}(\alpha(t), 0) & =T_{m}, \\
\theta_{2}(\infty, t) & =0
\end{aligned}
$$

and the Stefan's condition is as

$$
-\lambda_{1}=\frac{\partial \theta_{1}(\alpha(t), t)}{\partial r}=-\lambda_{2}=\frac{\partial \theta_{2}(\alpha(t), t)}{\partial r}+L \gamma \frac{d \alpha(t)}{d t} .
$$

Here we represent $\alpha(t)$ as follows

$$
\alpha(t)=b+\alpha_{1} t^{1 / 2}+\alpha_{2} t+\cdots+\alpha_{n} t^{n / 2}+\cdots,
$$

where $\alpha_{1}, \alpha_{2}, \alpha_{3}, \ldots$ have to be determined.

By making substitution $\theta=\frac{u}{r}+T_{m}$ and $r=x+b$ in (2)-(13) we reduce problem (2)-(12) to the following problem:

$$
\begin{aligned}
& \frac{\partial U_{1}}{\partial t}=a_{1}^{2} \frac{\partial^{2} U_{1}}{\partial x^{2}}, \\
& \frac{\partial U_{2}}{\partial t}=a_{2}^{2} \frac{\partial^{2} U_{2}}{\partial x^{2}},
\end{aligned}
$$




$$
\begin{aligned}
U_{1}(0,0) & =0, \\
U_{2}(x+b, 0) & =\left[f(x+b)-T_{m}\right](x+b), \\
f(0) & =T_{m}, \quad \beta(0)=0, \quad f(\infty)=0, \\
x & =0: \quad-\lambda_{1}\left[b \frac{\partial U_{1}}{\partial x}-U_{1}\right]_{x=0}=b^{2} P(t), \\
x & =\beta(t): \quad U_{1}(\beta(t), t)=0, \\
U_{2}(\beta(t), t) & =0, \\
U_{2}(\infty, t) & =0,
\end{aligned}
$$

subject to the Stefan's condition

$$
-\lambda_{1}\left[\beta(t) \frac{\partial U_{1}}{\partial x}-U_{1}\right]_{x=\beta(t)}=-\lambda_{2}\left[\beta(t) \frac{\partial U_{2}}{\partial x}-U_{2}\right]_{x=\beta(t)}+\beta^{2}(t) \frac{d \beta(t)}{d t} L \gamma,
$$

where

$$
\beta(t)=\alpha(t)-b=\alpha_{1} t^{1 / 2}+\alpha_{2} t+\ldots+\alpha_{n} t^{n / 2}+\ldots=\sum_{n=0}^{\infty} \alpha_{n+1} t^{(n+1) / 2} .
$$

We represent solution in the form

$$
\begin{aligned}
U_{1}(x, t)= & \sum_{n=0}^{\infty} A_{2 n}\left(2 a_{1} t\right)^{n}\left[i^{2 n} \operatorname{erfc} \frac{-x}{2 a_{1} \sqrt{t}}+i^{2 n} \operatorname{erfc} \frac{x}{2 a_{1} \sqrt{t}}\right] \\
& +\sum_{n=0}^{\infty} A_{2 n+1}\left(2 a_{1} t\right)^{\frac{2 n+1}{2}}\left[i^{2 n+1} \operatorname{erfc} \frac{-x}{2 a_{1} \sqrt{t}}-i^{2 n+1} \operatorname{erfc} \frac{x}{2 a_{1} \sqrt{t}}\right], \\
U_{2}(x, t)= & \sum_{n=1}^{\infty} B_{n}\left(2 a_{2} t\right)^{n / 2}\left[i^{n} \operatorname{erfc} \frac{-x}{2 a_{2} \sqrt{t}}\right]+\sum_{n=1}^{\infty} C_{n}\left(2 a_{2} t\right)^{n / 2}\left[i^{n} \operatorname{erfc} \frac{x}{2 a_{2} \sqrt{t}}\right] .
\end{aligned}
$$

Here coefficients $A_{2 n}, A_{2 n+1}, B_{n}, C_{n}$ have to be found. Moreover, it is necessary to find unknown moving $\beta(t)$. Using Hermite polynomials like in [7] we represent (24) in the form of heat polynomials:

$$
\begin{aligned}
U_{1}(x, t) & =\sum_{n=0}^{\infty} A_{2 n} \sum_{m=0}^{n} x^{2 n-2 m} t^{m} \beta_{2 n, m}+\sum_{n=0}^{\infty} A_{2 n+1} \sum_{m=0}^{n} x^{2 n-2 m+1} t^{m} \beta_{2 n+1, m}, \\
A_{2 n+1} & =A_{2 n} \frac{\beta_{2 n, n}}{b \beta_{2 n+1, n}}-\frac{b}{\lambda_{1} \beta_{2 n+1, n}} \frac{P^{(n)}(0)}{n !} .
\end{aligned}
$$

To find $A_{2 n}$ we use multinomial coefficients of Newton's Polynomials. Thus to derive recurrent formula for $A_{2 n}$, we take both sides of (19) $2 \mathrm{k}$ times derivative at $\tau=0$ and get following expressions:

$$
\begin{aligned}
0^{(4 l)}= & \sum_{n=1}^{l} A_{2 n} \sum_{m=0}^{n-1} C_{2 n, m}[4 l]+\sum_{n=l+1}^{2 l-1} A_{2 n} \sum_{m=0}^{2 l-n-1} C_{2 n, m+2(n-l)}+A_{4 l} \beta_{4 l, 2 l} \\
& +\sum_{n=1}^{l} A_{2 n+1} \sum_{m=0}^{n-1} C_{2 n-1, m}[4 l] \beta_{2 n-1, m} \\
& +\sum_{n=l+1}^{2 l} A_{2 n-1} \sum_{m=0}^{2 l-n} C_{2 n-1, m+2(n-l-1)}[4 l] \beta_{2 n-1, m+2(n-l)-1},
\end{aligned}
$$

where $l=1,2, \ldots$ and $A_{0}=0$.

$$
\begin{aligned}
0^{(2(2 l-1))}= & \sum_{n=1}^{l-1} A_{2 n} \sum_{m=0}^{n-1} C_{2 n, m}[2(2 l-1)] \beta_{2 n, m} \\
& +\sum_{n=l}^{2 l-1} A_{2 n} \sum_{m=1}^{2 l-n-1} C_{2 n, m+2(n-l)}[2(2 l-1)] \beta_{2 n, m+2(n-l)}+A_{4 l-2} \beta_{4 l-2,2 l-1}[2(2 l-1)]
\end{aligned}
$$




$$
\begin{aligned}
& +\sum_{n=0}^{l-1} A_{2 n+1} \sum_{m=0}^{n} C_{2 n+1, m}[2(2 l-1)] \beta_{2 n+1, m} \\
& +\sum_{n=l}^{2 l-1} A_{2 n+1} \sum_{m=1}^{2 l-n-1} C_{2 n+1, m+2(n-l)+1}[2(2 l-1)] \beta_{2 n+1, m+2(n-l)+1}
\end{aligned}
$$

Thus $A_{2 n}$, coefficients can be explicitly expressed from (28) and (29) where $C_{i, j}[4 l]$ or $C_{i, j}[4 l-2]$ multinomial coefficients or sums of coefficients at $\beta_{i, j}$.

By Lemma 1 and condition (16) we get following theorem.

Theorem 2 For $b \approx 0, g(x) \subset C^{\infty}$ we have

$$
B_{n}=\frac{1}{2} g^{(n)}(0),
$$

where

$$
g(x)=\left[f(x)-T_{m}\right] x .
$$

In similar manner in (20) we use Leibniz's formula, Faa Di Bruno's formula and Bell polynomials to calculate $C_{n}$ and get

$$
\begin{gathered}
\sum_{n=0}^{k} \frac{g^{(n)}(0)}{n !} \mu_{1}+\sum_{n=0}^{k} C_{n} \mu_{2}=0, \\
\mu_{1,2}=(2)^{1 / 2} \frac{k !}{(k-n) !} \sum_{m=1}^{k-n}( \pm 1)^{m} \frac{\Gamma\left(\frac{n-m+1}{2}\right)}{(n-m) ! \sqrt{\pi}} \sum \frac{(k-n) !}{j_{1} ! j_{2} ! \ldots j_{k-n-m+l} !} \beta_{1}^{j_{1}} \beta_{2}^{j_{2}} \ldots \beta_{k-n-m+1}^{j-k-n-m+l} .
\end{gathered}
$$

To calculate coefficients of $\beta(\tau)$ we use Stefan's condition (22).

By making substitution $\sqrt{t}=\tau$ and taking both sides of (21) k-times derivative at $\tau=0$ we reduce (22) to the following

$$
\left.\lambda_{2}\left[\frac{\partial^{k}\left[U_{2 x}(\beta(\tau), \tau) \sigma^{-1}(\tau)\right]}{\partial \tau^{k}}\right]\right|_{\tau=0}-\left.\lambda_{1}\left[\frac{\partial^{k}\left[U_{1 x}(\beta(\tau), \tau) \sigma^{-1}(\tau)\right]}{\partial \tau^{k}}\right]\right|_{\tau=0}=\beta_{k} \Lambda,
$$

where $\sigma(\tau)=\alpha_{1}+\alpha_{2} \tau+\alpha_{3} \tau^{2}+\ldots$ and $L \gamma=\Lambda$,

$$
U_{i x}(\beta(\tau), \tau)=\left.\frac{\partial U_{i x}}{\partial x}\right|_{x=\beta(\tau)},
$$

where $i=1,2$. Finally we get following recurrent formula from (34)

$$
\begin{aligned}
\beta_{k}= & \frac{\lambda_{2}}{\Lambda} \sum_{p=0}^{k}\left(\begin{array}{c}
k \\
p
\end{array}\right)\left\{\sum _ { n = 0 } ^ { \infty } \frac { 2 ^ { n / 2 } ( k - p ) ! } { ( k - p - n ) ! } \left[( - 1 ) ^ { n } B _ { n } \left(\sum_{m=1}^{k-p-n} i^{n-m} \operatorname{erfc} \alpha_{1}\right.\right.\right. \\
& \left.\sum \frac{(k-p) ! \beta_{1}^{j_{1}} \beta_{2}^{j_{2}} \ldots \beta_{k-p-n-m+1}^{j_{k-p-n+1}}}{j_{1} ! j_{2} ! \ldots j_{k-p-n-m+1} !}\right)+C_{n} \sum_{m=1}^{k-p-n} i^{n-m} \operatorname{erf} c\left(-\alpha_{1}\right) \\
& \left.\left.\left.\sum \frac{(k-p) ! \beta_{1}^{j_{1}} \beta_{2}^{j_{2} \ldots \beta_{k-p-n-m+1}^{j_{k-p-n+1}}}}{j_{1} ! j_{2} ! \ldots j_{k-p-n-m+1} !}\right)\right]\right\}\left\{\sum_{m=1}^{n}(-1)^{m} \frac{1}{\sigma^{m+1}} \sum \frac{n ! \beta_{1}^{j_{1}} \beta_{2}^{j_{2}} \ldots \beta_{n-m+1}^{j_{n-m+1}}}{j_{1} ! j_{2} ! \ldots j_{n-m+1} !}\right\} \\
& -\left.\frac{\lambda_{1}}{\Lambda}\left[\frac{\partial^{k}\left[U_{1 x}(\beta(\tau), \tau) \sigma^{-1}(\tau)\right]}{\partial \tau^{k}}\right]\right|_{\tau=0} .
\end{aligned}
$$




\section{Convergence}

Convergence of (24) and (25) can be proved as follows: Let $\beta\left(t_{0}\right)=\mu_{0}$ for any time $t=t_{0}$. Then the series

$$
\begin{aligned}
U_{1}(x, t)= & \sum_{n=0}^{\infty} A_{2 n}(2 t)^{n}\left[i^{2 n} \operatorname{erfc} \frac{-\mu_{0}}{2 \sqrt{t}}+i^{2 n} \operatorname{erfc} \frac{\mu_{0}}{2 \sqrt{t}}\right] \\
& +\sum_{n=0}^{\infty} A_{2 n+1}(2 t)^{\frac{2 n+1}{2}}\left[i^{2 n+1} \operatorname{erfc} \frac{-\mu_{0}}{2 \sqrt{t}}-i^{2 n+1} \operatorname{erfc} \frac{\mu_{0}}{2 \sqrt{t}}\right] \\
U_{2}(x, t)= & \sum_{n=1}^{\infty} B_{n}(2 t)^{n / 2}\left[i^{n} \operatorname{erfc} \frac{-\mu_{0}}{2 \sqrt{t}}\right]+\sum_{n=1}^{\infty} C_{n}(2 t)^{n / 2}\left[i^{n} \operatorname{erfc} \frac{\mu_{0}}{2 \sqrt{t}}\right]
\end{aligned}
$$

should be convergent, because $u_{1}=u_{2}=0$ on the interface. Therefore there exist some constants $C_{1}, C_{2}$, independent of $n$, such that

$$
\left|A_{2 n}\right|<C_{1} /\left(2 t_{0}\right)^{n}\left[i^{2 n} \operatorname{erfc} \frac{-\mu_{0}}{2 \sqrt{t_{0}}}+i^{2 n} \operatorname{erfc} \frac{\mu_{0}}{2 \sqrt{t_{0}}}\right] .
$$

Since $A_{2 n}$ are bounded and expressed in terms of $A_{2 n+1}$, then $A_{2 n+1}$ is also bounded.

Multiplying both sides of (35) by $(2 t)^{n}\left[i^{2 n} \operatorname{erfc} \frac{(-\beta(t))}{2 \sqrt{t}}+i^{2 n} \operatorname{erfc} \frac{\beta(t)}{2 \sqrt{t}}\right]$ and taking sum we obtain that

$$
\begin{aligned}
\sum_{n=0}^{\infty} A_{2 n}(2 t)^{n}\left[i^{2 n} \operatorname{erfc} \frac{(-\beta(t))}{2 \sqrt{t}}+i^{2 n} \operatorname{erfc} \frac{\beta(t)}{2 \sqrt{t}}\right] & <C_{1} \sum_{n=0}^{\infty} \frac{(2 t)^{n}\left[i^{2 n} \operatorname{erfc} \frac{(-\beta(t))}{2 \sqrt{t}}+i^{2 n} \operatorname{erfc} \frac{\beta(t)}{2 \sqrt{t}}\right]}{\left(2 t_{0}\right)^{n}\left[i^{2 n} \operatorname{erfc} \frac{-\mu_{0}}{2 \sqrt{t_{0}}}+i^{2 n} \operatorname{erfc} \frac{\mu_{0}}{2 \sqrt{t_{0}}}\right]} \\
& <C_{1} \sum_{n=0}^{\infty}\left(\frac{t}{t_{0}}\right)^{n} .
\end{aligned}
$$

In the same manner

$$
\begin{gathered}
\left|\quad C_{n}\right|<C_{2} /\left(2 t_{0}\right)^{n} i^{2 n} \operatorname{erfc} \frac{\mu_{0}}{2 \sqrt{t_{0}}} \\
\sum_{n=0}^{\infty} C_{n}(2 t)^{n}\left[i^{2 n} \operatorname{erfc} \frac{\beta(t)}{2 \sqrt{t}}\right]<C_{2} \sum_{n=0}^{\infty} \frac{(2 t)^{n}\left[i^{2 n} \operatorname{erfc} \frac{\beta(t)}{2 \sqrt{t}}\right]}{\left(2 t_{0}\right)^{n}\left[i^{2 n} \operatorname{erfc} \frac{\mu_{0}}{2 \sqrt{t_{0}}}\right]}<C_{2} \sum_{n=0}^{\infty}\left(\frac{t}{t_{0}}\right)^{n} .
\end{gathered}
$$

These are geometric series and the series for $u_{1}(x, t)$ converges for all $x<\mu_{0}$, while the series for $u_{2}(x, t)$ converges for all $x>\mu_{0}$ and $t<t_{0}$. The series $\beta(t)$ can be estimated similarly from the equation (35).

\section{CONCLUSION}

To summarize, the coefficients $A_{2 n+1}, A_{2 n}, B_{n}$ and $C_{n}$ are obtained from (27), (28), (29), (32), (33) and coefficients of free boundary $\beta(t)$ obtained from (35), and also convergence proved.

\section{ACKNOWLEDGMENTS}

This publication is supported by the target program 0085/PTSF-14 from the Ministry of Science and Education of the Republic of Kazakhstan. 


\section{REFERENCES}

1. R. Holm, Electric Contacts, Theory and Application, Springer-Verlag, Berlin, 1967, 4 edn.

2. S. N. Kharin, H. Nouri, and T. Davies, "The mathematical models of welding dynamics in closed and switching electrical contacts," in Holm Conf. on Electrical Contacts, IEEE, Washington, USA, 2003, pp. 107-123.

3. A. Friedman, J. Math. Mech 8, 499-517 (1959).

4. L. Rubinstein, The Stefan Problem, 1967.

5. S. N. Kharin, and M. Sarsengeldin, "Influence of contact materials on phenomena in a short electrical arc," in Key Engineering Materials, Trans tech publications, Islamabad, Pakistan, 2012, pp. 321-329.

6. A. N. Tikhonov, and A. A. Samarski, Equations of Mathematical Physics, Dover Publications, New York, 1963.

7. M. Sarsengeldin, and S. Kharin, Filomat (2016), in Press.

8. M. M.Sarsengeldin, S. Guvercin, and S. Kassabek, "Solution of inverse Stefan problem by Hartree functions method," in Journal of Physics, Confference series, IEEE, Jakarta, Indonesia, 2014. 